
\title{
$\begin{array}{ll}\text { Research Square } & \begin{array}{l}\text { Preprints are preliminary reports that have not undergone peer review. } \\ \text { They should not be considered conclusive, used to inform clinical practice, } \\ \text { or referenced by the media as validated information. }\end{array}\end{array}$ \\ The Probability Distribution of Maximum Temperature to Assess the Suitable Statistical Models: Take the North-East and Southern Regions of Pakistan
}

Tasir khan ( $\nabla$ tasirkhan.state@gmail.com )

Lanzhou University https://orcid.org/0000-0003-0651-0418

\section{Yejuan Wang}

Lanzhou University

\section{Research Article}

Keywords: Probability distribution function, Kumaraswamy distribution, Statistical analysis, temperature data, Maximum likelihood estimator

Posted Date: July 14th, 2021

DOl: https://doi.org/10.21203/rs.3.rs-568429/v1

License: (c) (1) This work is licensed under a Creative Commons Attribution 4.0 International License.

Read Full License 
The probability distribution of maximum temperature to assess the suitable statistical models: Take the north-east and southern regions of Pakistan

3 Tasir Khan ${ }^{\mathrm{a}}$, , Yejuan Wang ${ }^{\mathrm{a}}$ *

$4 \quad{ }^{\text {a }}$ School of Mathematics and Statistics, Gansu Key Laboratory of Applied Mathematics

5 and Complex Systems, Lanzhou University, Lanzhou 730000, China

6

7

37 School of Mathematics and Statistics

38

39

40
*Corresponding author Email:

Dr. Tasir khan

Lanzhou University, Lanzhou 730000, China

tasirkhan.state@gmail.com 


\section{The probability distribution of maximum temperature to assess the suitable statistical} models: Take the north-east and southern regions of Pakistan

\section{Abstract}

Precise maximum temperature probability distribution information is indeed of accurately significance for numerous temperature uses. The purpose of this research to assess the appropriateness of these functions likelihood for evaluating the temperature models at different sites in southern part of Pakistan. The Kumaraswamy distribution function is used initially to approximation the models of maximum temperature. Compare the presentation of the Kumaraswamy distribution with twelve commonly used the probability functions. The consequences obtained show that the more effective functions are not similar across all sites. The maximum temperature features, quality and quantity of the noted temperature observation can be regarded as a factors that affect the presentation of the function. Similarly, the skewness of the noted maximum temperature observations may affect the precision of Kumaraswamy distribution. For the Hyderabad, Lahore and Sialkot sites, the Kumaraswamy distribution obtainable the topmost presentation, however for the Karachi, Multan stations, the generalized extreme value (GEV) distributions provided the best fit, respectively. According to the calculations, the Kumaraswamy distribution usually be regarded as a valid distribution because it runs 3 best fit sites and ranks 2 to 3 among the remaining sites. Though, the tight presentation of the Kumaraswamy and GEV and the flexibility of the Weibull distribution which has been usually verified, more evaluations of the presentation of the Kumaraswamy_distribution are needed.

Key words; Probability distribution function; Kumaraswamy distribution; Statistical analysis; temperature data; Maximum likelihood estimator

\section{Introduction}

Temperature evaluation shows an important role in reviewing continuous variations in temperature falls or rise. Many different works have been carried out in response to environmental climate change, and conclusions about the critical and difficult fall and rise of ambient temperature have been drawn (Karpouzos et al., 2010; Cui et al., 2017) (Huang et al., 2019). In case of weather variation or global temperature, small changes in the usual temperature value can cause major 
changes in the scale and frequency of extreme measures, with high temperatures (Yan et al.,

2 2016).According to the fifth valuation report of the Intergovernmental Panel on Weather Change, from 1880 to 2012 , the global average surface temperature increased by $0.85^{\circ} \mathrm{C}$. There is no doubt that global warming has had a serious influence on human society(Chen et al., 2019, Zhou et al.,

5 2017). As the temperature rises, the ability to retain water in the atmosphere increases, and the temperature of extreme value has changed significantly, leading to forest fires and frequent droughts. At the same time, with temperature changes, the global usual ocean level rise by $19 \mathrm{~cm}$ between 1900 and 2011. In other words, changes in extreme temperatures increase the intensity and frequency of extreme weather events (such as extreme temperatures, heavy rains, droughts, and floods. When the temperature rises, the desert will increase, and the infiltration and soil moisture intensity will change. The change in water rotation is caused by an increase in temperature. Rising temperature will also change the restructuring of river excess and the characteristics of water resources in the basin. Extreme temperature events is the main measure of extreme climate events. Therefore, the study of extreme temperature changes under global warming is great significance.

A current analysis of the detection of extreme temperature trends in Europe (Irannezhad et al., 2019) confirmed that 20th century over time, the warmth of day and night. Also concluded that the frequency and intensity of high temperature (low temperature) (Shaby and Reich, 2012, Parey et al., 2013, Naveau et al., 2014, Huang et al., 2016).Since the temperature probability, it is a feasible method to predict extreme precipitation based on the relationship between temperature and extreme precipitation under weather change. Therefore, in all over the world is studying the basic process of detecting extreme precipitation changes with temperature (Donat et al., 2016, Gao et al., 2018).(Wang et al., 2017) found that, compared with the historical temperature dependency of extreme precipitation, the peak temperature of extreme precipitation will rise with climate warming, which means that the peak arrangement does not mean the probable higher limit of extreme precipitation in the future. As mentioned above, the relationship between extreme 27 precipitations and has been fully studied, but the association among highest extreme rainfall and highest temperature (Teshome and Zhang, 2019).

All selection periods fit the GEV distribution and estimate the parameter. The likelihood ratio test shows that the best model in which the position parameter increases linearly, and the parameters shape and scale are constant. Model diagnosis including quantile plots, probability 
1 plots and density plot showed a good degree of fit. The GOF test Anderson Darling and

2 Kolmogorov Simonov show that modeling can almost gave same fitting result (Hasan et al., 2012,

3 Hughes et al., 2007). A univariate extreme value (EV) model based on the limit temperature data

4 of the block maximum method. The block size selection is important because when size is large,

5 valuable information may be wasted. Using a block length of one year will generate too few maximum sequences (20 data) and result in a higher estimate variance. However, a block length that is too short will not meet the limit approximation of extreme value temperature (EVT).

8 Therefore, the length is half-yearly, quarterly and monthly blocks are still feasible. From the analysis of quantile graph, monthly and annual blocks are more suitable than quarterly and semiannual blocks. Generally, LM is better than MLE in estimating parameters. Numerical results indicate that the maximum temperature will gradually increase (Amin et al., 2018).

The absolute burden of heat waves that is not conducive to health. This is in the entire community, but workers who work in various hot places are particularly vulnerable. Therefore, the impact is also economical. Since this growing hazard, the health authorities of the Republic of Djibouti would play an important role conducting research on the actual impact of high temperatures on morbidity and mortality, and promoting, leading and evaluating a series (Ozer and Mahamoud, 2013). During the confirmation period statistical downscaling model (SDSM) obtained better results from monthly seasonal and daily time sequence. In other words, the results based on the seasonal sequence are slightly better than the monthly sequence. The performance of this model is suitable for SDSM when trying to simulate future extreme temperature indexes. In the verification process, the intensity limit index is better than the frequency index (Mahmood and distribution. Under simulated warmer weather situations, the temperature change (standard deviation of the distribution) in summer is relatively unchanged, but in winter (especially in higher latitudes) it is significantly reduced (Holmes et al., 2016). The extreme temperature increase occurred in the last ten years. The influence of temperature is the cause of changing Pakistan's climate. For example, heat waves are increasing across the country (Afzaal et al., 2009). Accurately estimating the long-term trends of global and regional climate change are essential for the impact and prediction attributable to climate change (Li et al., 2020, Li et al.).

The objective of this study due to the effective application of the Kumaraswamy distribution in different fields, it may be interesting to evaluate its proficiency in the designated 
case study to estimate the maximum temperature distribution. Thus, the presentation of the

2 Kumaraswamy distribution was tested for certain before used distribution functions (including exponential, normal, invers-Gaussian, logistic, log-logistic, log-normal 3, Gumbel Generalize

4 Extreme Value, Weibull 3, Pearson type 3, and Generalize-Gamma distribution. Statistical 5 evaluation of the efficiency of all twelve distribution functions based on broadly used statistical parameters. To determine the most suitable theoretical function by using the In addition, KS, AD, AIC and BIC are used as goodness of fit indicators.

8 Methodology

$9 \quad$ Probability distribution modeling of maximum temperature

10 Generally, understanding the probability distribution of maximum temperature is essential for 11 characterizing temperature behavior, evaluating maximum temperature performance. Therefore, it 12 is important to determine the most suitable function for temperature data. In this study, twelve 13 PDFs were used to describe the frequency distribution of temperature. These distribution functions 14 are exponential, N, IG,L, LL3, LN3, GUM, KUM, GEV, WEI3, PE3, GG Weibull, gamma, 15 lognormal, Gaussian inverse, logarithmic, generalized extreme and upper middle. In this study, the 16 Kum distribution is used for the first time to describe the maximum temperature distribution in the 17 selected case study. MLE is used to estimate the parameters that define each PDF. MLE is 18 generally regarded as a very reliable technique, which can determine the parameter value that maximizes the likelihood of the data used [41]. For more sample sizes, MLE is most efficient than estimate approaches such as the Linear moments (LMOM), method of moments (MOM) and

21 produces a lesser mean square error.

\section{Table 1}

23 Appraising the fitting performance

24 The special probability distribution is affected by a number of aspects, therefore the PD parameter 25 evaluation methods, comparison methods and the availability of rainfall data. In this research, chi 26 square and KS test was used to assess the fitness of certain PDs. The KS and chi square test 27 calculated test statistics that define the theoretical value and actual value estimate from distributions. In addition, in order to check the visual estimation of the goodness of fit. The advantage of the above test plot and fit test applied to the maximum rainfall data (Morgan et al., 
2009, Lollchund et al., 2014, Cassalho et al., 2018, Fawad et al., 2019). Weather test statistics and

2 fitted test described above can consistently use to select the best fit distribution.

3 In order to evaluate the effectiveness checked PDF for modeling the probability distribution of 4 temperature, statistical displays of $\mathrm{AD}, \mathrm{KS}$, Bayes Information Criterion (BIC) and Akaike 5 Information Criterion (AIC) are used . Statistically used to examine the deviation among the

6 forecast data and the observed data using a probability function. These indicators are briefly

7 introduced here [43]

8 The KS statistics is usually based on the empirical distribution function (EDF), and the sample comes from continuous distribution. A random sample assumes that there are $x_{1}, x_{2}, x_{3}, \ldots, x_{n}$ in a certain distribution. Then you can define EDF in the following ways

$$
F_{n}(x)=\frac{1}{n}[\text { number of observation } \leq x]
$$

12 The KS test the theoretical probability distribution as

$$
D=\max _{1 \leq i \leq n},\left[F\left(x_{i}\right)-\frac{i-1}{n}, \frac{i}{n}-F\left(x_{i}\right)\right]
$$

In equation (2), $F\left(x_{i}\right)$ is the cumulative distribution function, $x_{i}$ is the $i^{\text {th }}$ order statistic and $\mathrm{n}$ denote the sample size.

The Anderson Darling (A-D) test associates the observed fitting of the distribution. The Anderson darling test assigns a higher weighted distribution to the tail, (Fawad et al., 2019) . The A-D statistics $A^{2}$ test as:

$A^{2}=-n-\frac{1}{n} \sum_{i=1}^{n}(2 i-1)\left[\ln F\left(x_{i}\right)+\ln \left(1-F\left(X_{n-i+1}\right)\right)\right]$

Where $A^{2}$ relates to the test result, $\mathrm{X}$ is the variable, $F\left(X_{i}\right)$ is the distribution function, and $\mathrm{n}$ is the sample size and uses the statistical model with the smallest AD score for the data of wind speed as the most-fitting distribution model.

\section{Study Area}

24 Pakistan's climate has many unique cyclical changes. The most serious variables affecting the climate are humidity, temperature, wind speed and rainfall. The deserts in some areas are still very hot and dry.

27 Karachi is the most populous city in Pakistan and the capital of Sindh Province. Karachi is located in southern Pakistan in Sindh Province. Therefore, the summer is not hot and humid from December to February. Compared with the hot season that started in March and lasted until the June monsoon, it was dry and pleasant. Hyderabad is also located in Sindh province, the desert 
climate in Hyderabad is hot and warm throughout the year. This city is famous for its tempering the originally hot climate. As a result, houses in Hyderabad have traditionally been equipped with "induction wind" towers that blow breeze in to residential areas to reduce heat. From mid-April to late June is the hottest period of the year, with the highest peak in May at $41.4^{\circ} \mathrm{C}$. The maximum

5 temperature recorded on May was $50^{\circ} \mathrm{C}$, and the lowermost temperature recorded on February was $6 \quad 1^{\circ} \mathrm{C}\left(34^{\circ} \mathrm{F}\right)$.

7 Lahore, the main city and cultural and historical midpoint of Punjab. The weather in Lahore is 8 semi-arid. In June, the rainy season begins. The temperature rises in July.

9 Sialkot is located in Panjab in the northwest. It has four sub seasonal humid and subtropical characteristics. The weather in Sialkot is still hot during the day, but cool at night and low humidity. In winter, the climate is a bit warm and there is a lot of precipitation. Multan is located in the southern part of Punjab and has witnessed the most extreme temperatures in Pakistan. Multan has an arid climate, with hot summers and cold winters. Summer begins in May and lasts until September. In Multan, summer is the longest season, while in the monsoon season there is heavy rain.

This article will use all of the above five stations to characterize the maximum temperature features. The maximum temperature sequence of weather sites Hyderabad, Karachi, Lahore, Multan and Sialkot are composed from the Pakistan Meteorological Department (PMD). Thirtysix years of data will be used to find the possible trends in the highest temperature. The AMT data from 1981 to 2016 will be analyzed in several years. The locations of the eight stations are given 21 in Figure 1.

Fig. 1. Map of five selected locations in Pakistan

Table 2. Descriptive analysis of five stations

\section{Results and discussion}

In this research, different distribution the goodness of fit test is evaluated to describe the distribution of temperature at five areas in the north-east and southern of Pakistan. In this affection, the Kum distribution is 1st time and previously related with some distribution.

31 In Table 2 these stations are measured descriptive of the entire database. Gives a detailed

32 description of the selected sites, which contains information about the statistical characteristics of 
1 the recording time, geographic location and temperature data. Some descriptive statistics,

2 including the maximum temperature, standard deviation, mean, skewness, and kurtosis used by

3 the selected sites. Table 3 displays all statistical distributions functions of Hyderabad, Karachi,

4 Lahore, Multan and Sialkot stations. In table 3 last column shows the rank of separately

5 distribution function. It should be noted that based on all numerical parameters, Kum is determined

6 to be the most appropriate distribution for Hyderabad, Sialkot and Lahore sites. However, for

7 Karachi and Multan stations the GEV distribution functions run the best fit to measure temperature

8 data. For Hyderabad, Karachi, Lahore, Multan and Sialkot stations, the parameters to obtain the

9 best distribution function are Kum $\left(\alpha_{1}=1.9422 \alpha_{2}=1.4754 a=21.301 b=43.15\right), \quad G E V(k=-$

$10 \quad 0.61938 \sigma=3.5027 \mu=31.764) \quad$ Kum $\left(\alpha_{1}=1.8367 \quad \alpha_{2}=1.6086 a=14.834 b=43.175\right)$, GEV $\quad(k=-$

$110.47692 \sigma=7.985 \mu=30.561)$ and Kum ( $\left.\alpha_{1}=1.8116 \alpha_{2}=1.5544 a=13.266 b=42.257\right)$. The

12 consequences also express that, exclude for Hyderabad site, the Wei3 function can operate

13 normally in other stations, so it can be used as the third or fourth best distribution. In addition, the

14 poorest performance showed that Exponential distribution represents its lowest priority.

15 The main results from table 3 is more effective approaches which are not same between sites. About parameters such as temperature features, the quality and quantity of noted the temperature

17 data may affect the validity of the distribution function to denote the temperature distribution to 18 check the location. Further, the results indicate that skewness may be the main parameter affecting the accuracy of Kum model. It can be seen that the Kum distribution ranks first in the Lahore, Sialkot and Multan sites with lower skewness, whereas it indicate that lower efficiency for other sites with greater skewness (see table 2 and 3 association). However, in future studies, the effects of all the above parameters must be properly studied to draw conclusions.

\section{Table 3}

Additional outcome displays that for all five sites, the Gam model indications comparative flexibility for all temperature data and ranks in the top four in expressions of proficiency. In addition, the Kum, Wei3 and GEV distribution models are most flexible because they can provide that the Kum distribution is usually measured an effective function because it offers the most 
suitable in 3 sites, and it ranks 3rd to 5th among the remaining 3 sites. However, due to the tightness

2 of the Kum and Wei distribution functions and the flexibility of the Wei function as its broadly verified characteristics in previous studies, the efficiency of the Kum distribution should be examined more in future studies. For this reason, more situation studies with different temperature

5 features must be estimated. In order to illustrate that the four most suitable distribution function describe the temperature in different ranges, figure 2(a-e) shows that CDF and PDF and curves fitted by all stations. For pdf and cdf graphs, the horizontal axis is the range of temperature data. For pdf plots, the shows the probability density, which varies between the highest and lowest probable value. For the cdf graph, the perpendicular axis shows the cumulative density, as we move from left to right on the parallel axis, the value increase from 0 to 1 .

Fig. 2. Cumulative distribution (CDF) and Histograms with fitted distributions for: (a) Hyderabad (b) Karachi (c) Lahore (d) Multan (e) Sialkot

The precise return level of extreme temperature must be estimate. The GEV distribution is used to fit extreme temperature sequence of each site. The change position series is installed in the station with the variation point (Yan et al., 2016). Dort and David (2016) a consistent method was used to examine the modulation of the extreme probability of temperature and rainfall, and it was found that the extreme maximum temperature has a statistically significant long-term increase, but has obvious seasonal and regional changes. They used the Wilcoxon test and Boxplots summarized the results of four AEPs $(50 \%, 10 \%, 5 \%$, and 1\%) and fixed and non-fixed generalized extreme value (GEV) distributions. The occurrence of temperature waves between 1985 and 2005 in north Pakistan and the fast melting of glaciers proved the increasing trend of weather warming in Pakistan (Rasul et al., 2008). A bivariate stochastic model for the space time field of maximum and minimum temperature. The bivariate field splits in to two parts "weather" and "local climate". The climate factor is spatially related to bivariate simulation. The statistical model adds the blocking effect of spatial variation to allow small scale variability of local variation model and successfully adapts to the stationarity of cross covariance and direct covariance functions over time.(Kleiber et al., 2013).

The estimator AIC is the prediction error of sample, and therefore the comparative superiority of the statistical model for data set. (McElreath, 2020, Taddy, 2019) given the set of models used for 
the data, AIC is the quality of estimations of individually model relative to every model. Therefore,

2 AIC provides a method of model selection. AIC is based on particular theory, when using a statistical model denote the process of generating data, the representation is almost certainly not accurate. Therefore, a model to represent the procedure will lose some information. AIC

5 estimations the comparative amount of data missing a specified model, when assessing the total data lost, AIC will weigh the models goodness-of-fit and model simplicity. For identification of mode the application of BIC widely used in linear regression and time series. However, it can be widely applied to models based on maximum likelihood. Because the interested model is equal to number of parameters.

The other method is based on the relative measure of information loss when fitting the model to describe the data. This approach includes Akaike information criteria AIC and BIC. However, these two technique is the most popular measure. In the sense of hypothesis testing, AIC is not a model test. Rather, the process and scoring provide a method for comparing data models and a tool

14 for model selection. The general formula for AIC and BIC is

$15 \quad \mathrm{AIC}=-2 \log (L)+2 k$

$\mathrm{BIC}=-2 \log (L)+\mathrm{k} \log (\mathrm{n})$

17 Where $\mathrm{K}$ is the number of parameter and $\mathrm{L}$ is likelihood of the fitted model [74-75]. These criteria take in to account the simplicity of the model because they contain penalties that increase with the number of parameters. AIC and BIC penalized the logarithmic probability criterion, thereby maintaining a balance between good fit and complexity. The model selection of the best fit

\section{Conclusion}

In this research, the efficiency of different function was estimate to denote the function of maximum temperature at 5 sites in the Pakistan.in this study, a new function called kumarsawamy (Kum) was estimated first time. MLE is an actual parameter estimation method used to analyze the related parameters. The results shows that is impossible to present one appropriate distribution for all check sites. For Hyderabad, Lahore and Sialkot sites, the Kumaraswamy distribution was found to be most suitable for maximum temperature data, while for Karachi and Multan stations GEV functions is most appropriate. It was originate that certain parameters such as maximum temperature features, the quality and quantity of noted temperature data can be measured the 
1 distribution as an effect on the performance. In addition, skewness is the main parameter that

2 affects the precision of the Kumaraswamy distribution, so it ranks first in the Hyderabad, Lahore and Sialkot stations with lower skewness, and shows lower efficiency for other sites with higher

4 skewness. However, in the future research, the impact of the above revealed important parameters

5 of the function should be properly studied to draw conclusions. For all sites the results show that

6 Kum, Wei3, and GEV are more flexible in distribution because they can display better 7 performance.

8 Generally, this research shows that the Kum distribution function is an actual distribution because

9 it runs the most fit in 2 stations, and it ranks 2 nd among the remaining 2 stations. However, due to the 10 tightness of the Kum, GEV Wei3 distribution functions and the flexibility of the GEV and wei3 function 11 as its widely proven characteristics in previous studies, the effectiveness of the Kum distribution should be 12 evaluated more in future study. For this reason, more situation study with different temperature features 13 would also be estimated.

\section{Acknowledgments}

15 We thank our respected reviewers and especially Yejuan Wang for their valuable comments and 16 suggestions that helped us to improve this paper.

\section{Conflict of interest}

18 The authors declare that they have no conflict of interest

Funding. No

\section{Author's contribution}

21 All authors contributed to the study conception and design. Material preparation, data collection 22 and analysis were performed by [Tasir khan] and [yejuan wang]. The first draft of the manuscript was written by [Tasir khan] and all authors commented on previous versions of the manuscript. All authors read and approved the final manuscript.Tasir Khan. Data curation, Writing- Original 25 draft preparation. Yejuang wang; Supervision:

\section{Data availability statement}

27 All the authors of this manuscript confirmed that the data supporting the findings of this study are available in the article. All the required data is available and easily accessible. 
1

2

3

4

5

6

7

8

9

10

11

12

13

14

15

16

17

18

19

20

21

22

23

24

25

26

27

28

29

30

31

32

33

34

35

36

37

38

39

40

41

42

43

44

45

\section{https://www.rstudio.com/products/rstudio/download/}

https://easyfit.en.softonic.com/

\section{Pakages}

L-mom

$\underline{\text { lmomco-package }}$

L-Moments

data(groundbeef)

serving <- groundbeef\$serving

(fitg <- fitdist(serving, "gamma"))

gofstat(fitg)

(fitln <- fitdist(serving, "lnorm"))

gofstat(fitln)

gofstat(list(fitg, fitln))

data(toxocara)

number <- toxocara\$number

fitp <- fitdist(number, "pois")

summary(fitp)

plot(fitp)

fitnb <- fitdist(number,"nbinom")

summary(fitnb)

plot(fitnb)

set.seed(1234)

$\mathrm{x} 4<-$ rweibull $(\mathrm{n}=1000$, shape $=2$, scale $=1)$

\# fit of the good distribution

f4 <- fitdist(x4,"weibull")

\# fit of a bad distribution

f4b <- fitdist(x4,"cauchy")

gofstat(list(f4,f4b),fitnames=c("Weibull", "Cauchy"))

lmoments $<$-Lmoments $(\mathrm{x})$;

$\operatorname{lmomcov}<-\operatorname{Lmomcov}(\mathrm{x})$;

estim_params<-1mom2normpoly4(lmoments);

hist(x,30,freq=FALSE)

plotpoints<-seq $(\min (\mathrm{x})-1, \max (\mathrm{x})+1, \mathrm{by}=0.01)$; 


1

lines(plotpoints,dnormpoly(plotpoints,estim_params),col='red');

lines(plotpoints,dnormpoly(plotpoints,true_params),col='blue');

\section{Consent for publication}

All the authors agree to publish this paper

\section{Ethical statement}

All experimental procedure were approved by the animal welfare and ethics committee of Lanzhou University (LZU-201805-224)

\section{Consent to participate}

This study involve no living organisms or their products so don't need any consent of participate

12

(4)

\section{References}

AMIN, N. A. M., ISMAIL, M. S. \& HAMID, H. A. Modelling extreme temperature in Perlis using block maxima method. AIP Conference Proceedings, 2018. AIP Publishing LLC, 020010.

CASSALHO, F., BESKOW, S., DE MELLO, C. R., DE MOURA, M. M., KERSTNER, L. \& ÁVILA, L. F. 2018. At-site flood frequency analysis coupled with multiparameter probability distributions. Water resources management, 32, 285-300.

CHEN, T., AO, T., ZHANG, X., LI, X. \& YANG, K. 2019. Climate change characteristics of extreme temperature in the Minjiang river basin. Advances in Meteorology, 2019.

DONAT, M. G., LOWRY, A. L., ALEXANDER, L. V., O'GORMAN, P. A. \& MAHER, N. 2016. More extreme precipitation in the world's dry and wet regions. Nature Climate Change, 6, 508-513.

FAWAD, M., YAN, T., CHEN, L., HUANG, K. \& SINGH, V. P. 2019. Multiparameter probability distributions for at-site frequency analysis of annual maximum wind speed with L-Moments for parameter estimation. Energy, 181, 724-737.

GAO, X., ZHU, Q., YANG, Z., LIU, J., WANG, H., SHAO, W. \& HUANG, G. 2018. Temperature dependence of hourly, daily, and event-based precipitation extremes over China. Scientific reports, 8, 1-10.

HASAN, H., RADI, N. A. \& KASSIM, S. Modeling of extreme temperature using generalized extreme value (GEV) distribution: A case study of Penang. World Congress on Engineering, 2012. 181-186.

HOLMES, C. R., WOOLLINGS, T., HAWKINS, E. \& DE VRIES, H. 2016. Robust future changes in temperature variability under greenhouse gas forcing and the relationship with thermal advection. Journal of Climate, 29, 2221-2236.

HUANG, W. K., STEIN, M. L., MCINERNEY, D. J., SUN, S. \& MOYER, E. J. 2016. Estimating changes in temperature extremes from millennial-scale climate simulations using generalized extreme value (GEV) distributions. Advances in Statistical Climatology, Meteorology and Oceanography, 2, 79-103.

HUGHES, G. L., SUBBA RAO, S. \& SUBBA RAO, T. 2007. Statistical analysis and time-series models for minimum/maximum temperatures in the Antarctic Peninsula. Proceedings of the Royal Society A: Mathematical, Physical and Engineering Sciences, 463, 241-259. 
IRANNEZHAD, M., MORADKHANI, H. \& KLØVE, B. 2019. Corrigendum to "Spatio-temporal Variability and Trends in Extreme Temperature Events in Finland over the Recent Decades: Influence of Northern Hemisphere Teleconnection Patterns". Advances in Meteorology, 2019.

KLEIBER, W., KATZ, R. W. \& RAJAGOPALAN, B. 2013. Daily minimum and maximum temperature simulation over complex terrain. The Annals of Applied Statistics, 588-612.

LOLLCHUND, R. M., BOOJHAWON, R. \& RUGHOOPUTH, S. D. 2014. Statistical modelling of wind speed data for Mauritius. International Journal of Renewable Energy Research (IJRER), 4, 1056-1064.

MAHMOOD, R. \& BABEL, M. S. 2014. Future changes in extreme temperature events using the statistical downscaling model (SDSM) in the trans-boundary region of the Jhelum river basin. Weather and Climate Extremes, 5, 56-66.

MCELREATH, R. 2020. Statistical rethinking: A Bayesian course with examples in $R$ and Stan, CRC press.

MORGAN, E., LACKNER, M., VOGEL, R. \& BAISE, L. 2009. Probability distributions for offshore wind speeds. AGUFM, 2009, A31F-0179.

NAVEAU, P., GUILLOU, A. \& RIETSCH, T. 2014. A non-parametric entropy-based approach to detect changes in climate extremes. Journal of the Royal Statistical Society: Series B: Statistical Methodology, 861-884.

OZER, P. \& MAHAMOUD, A. 2013. Recent extreme precipitation and temperature changes in Djibouti City (1966-2011). Journal of Climatology, 2013.

PAREY, S., HOANG, T. T. H. \& DACUNHA-CASTELLE, D. 2013. The importance of mean and variance in predicting changes in temperature extremes. Journal of Geophysical Research: Atmospheres, $118,8285-8296$.

SHABY, B. A. \& REICH, B. J. 2012. Bayesian spatial extreme value analysis to assess the changing risk of concurrent high temperatures across large portions of European cropland. Environmetrics, 23, 638-648.

TADDY, M. 2019. Business data science: Combining machine learning and economics to optimize, automate, and accelerate business decisions, McGraw Hill Professional.

TESHOME, A. \& ZHANG, J. 2019. Increase of extreme drought over ethiopia under climate warming. Advances in Meteorology, 2019.

WANG, G., WANG, D., TRENBERTH, K. E., ERFANIAN, A., YU, M., BOSILOVICH, M. G. \& PARR, D. T. 2017. The peak structure and future changes of the relationships between extreme precipitation and temperature. Nature Climate Change, 7, 268-274.

YAN, B., XIA, Z., HUANG, F., GUO, L. \& ZHANG, X. 2016. Climate change detection and annual extreme temperature analysis of the amur river basin. Advances in Meteorology, 2016.

ZHOU, B., XUE, H. \& SHANG, G. 2017. Characteristics of extreme climate in downstream catchment of Yangtze River during 1960-2012. Water Power, 43, 26-30. 


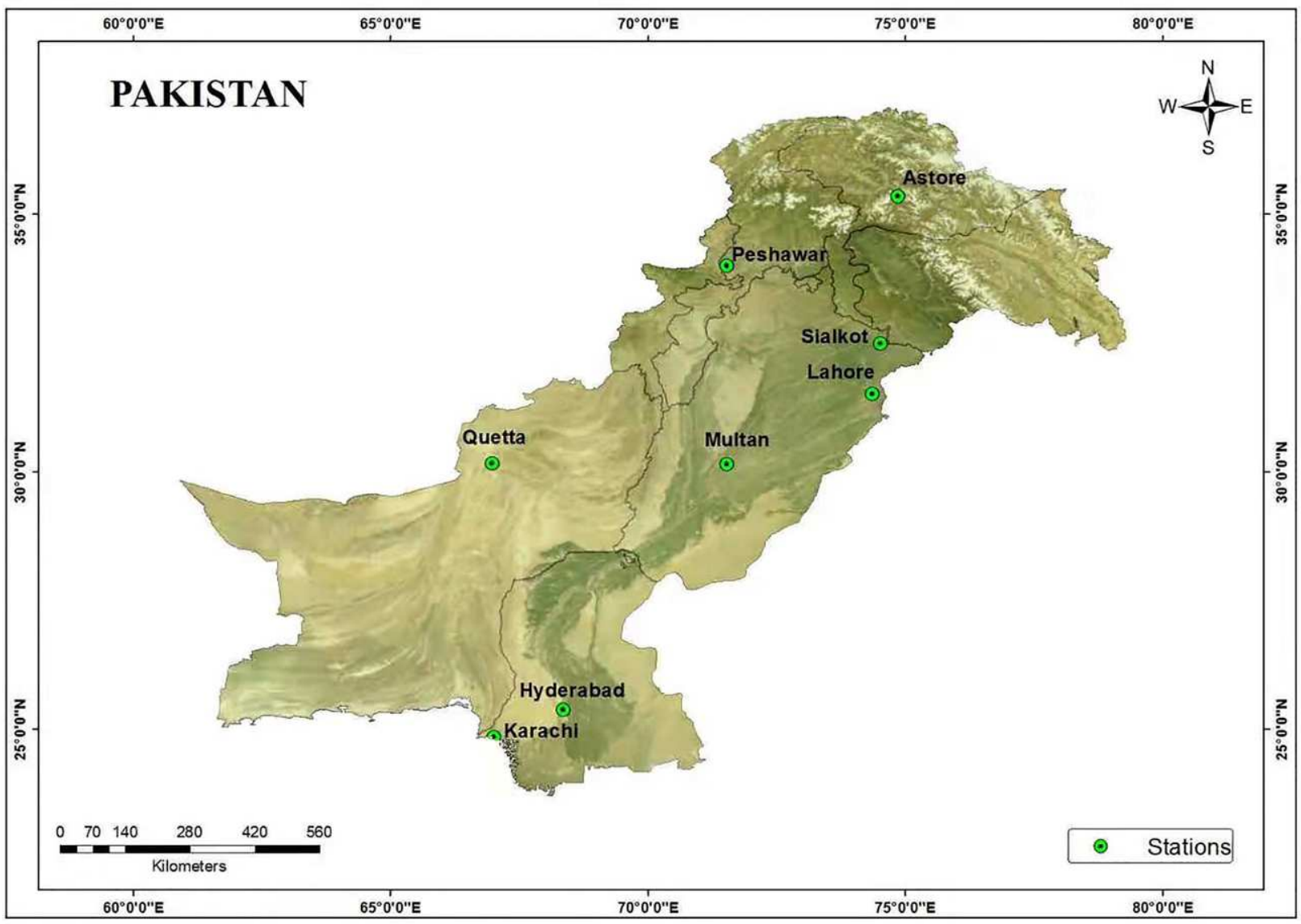

2 Fig. 1. Map of five selected locations in Pakistan

3

4

5

6

7 
Probability Density Function
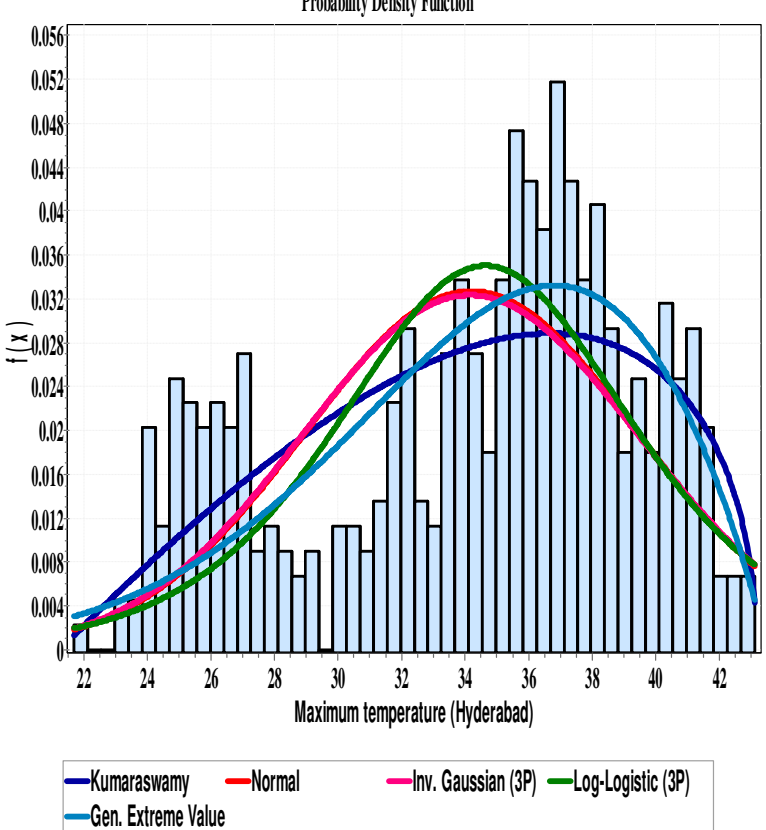

1

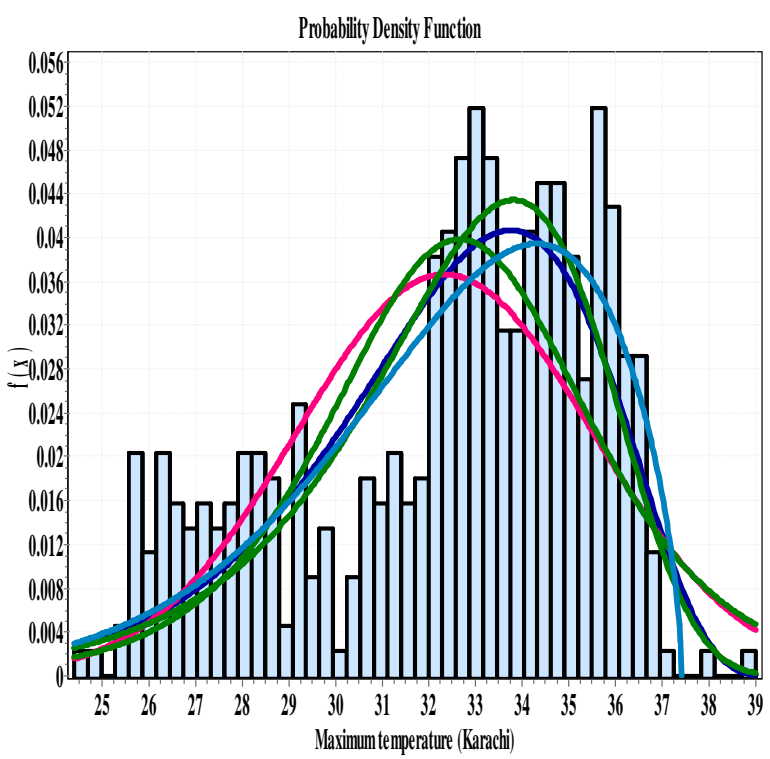

- Gen. Extreme Value

-Kumaraswamy -Inv.Gaussian (3P) -Log.Logistic (3P) -Weibull (3P)

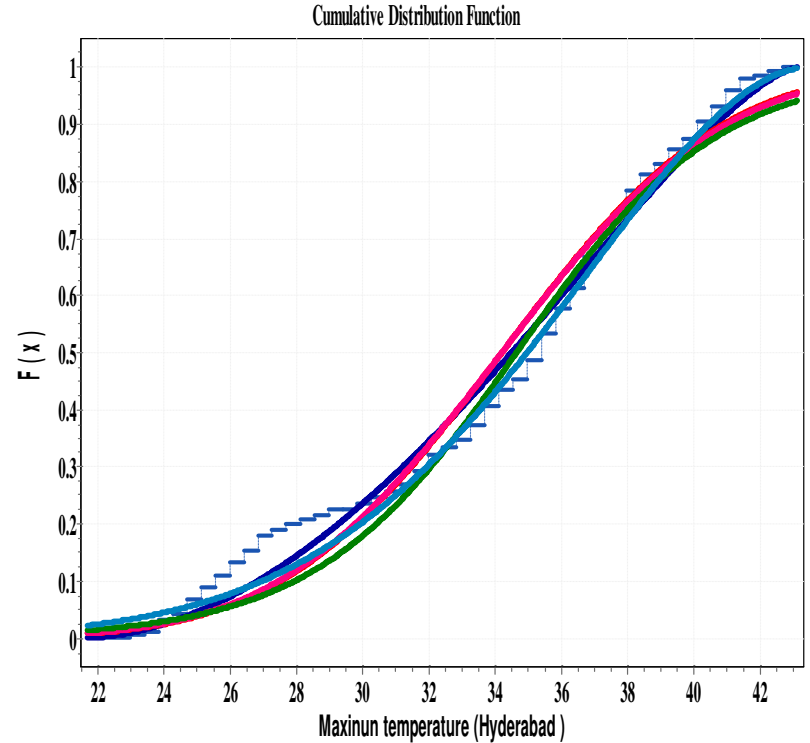

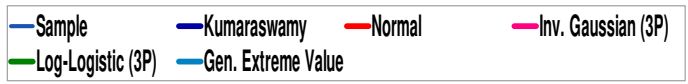

(a)

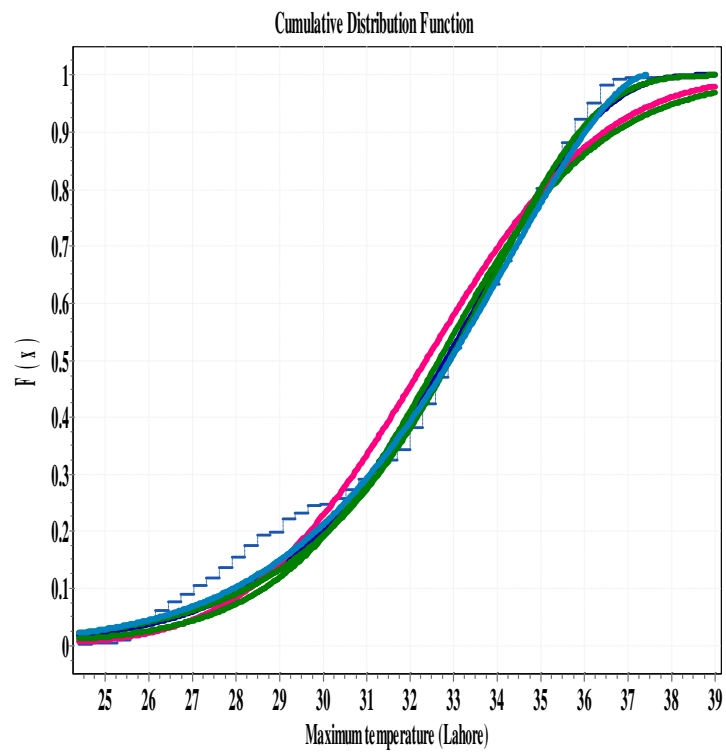

(b) 

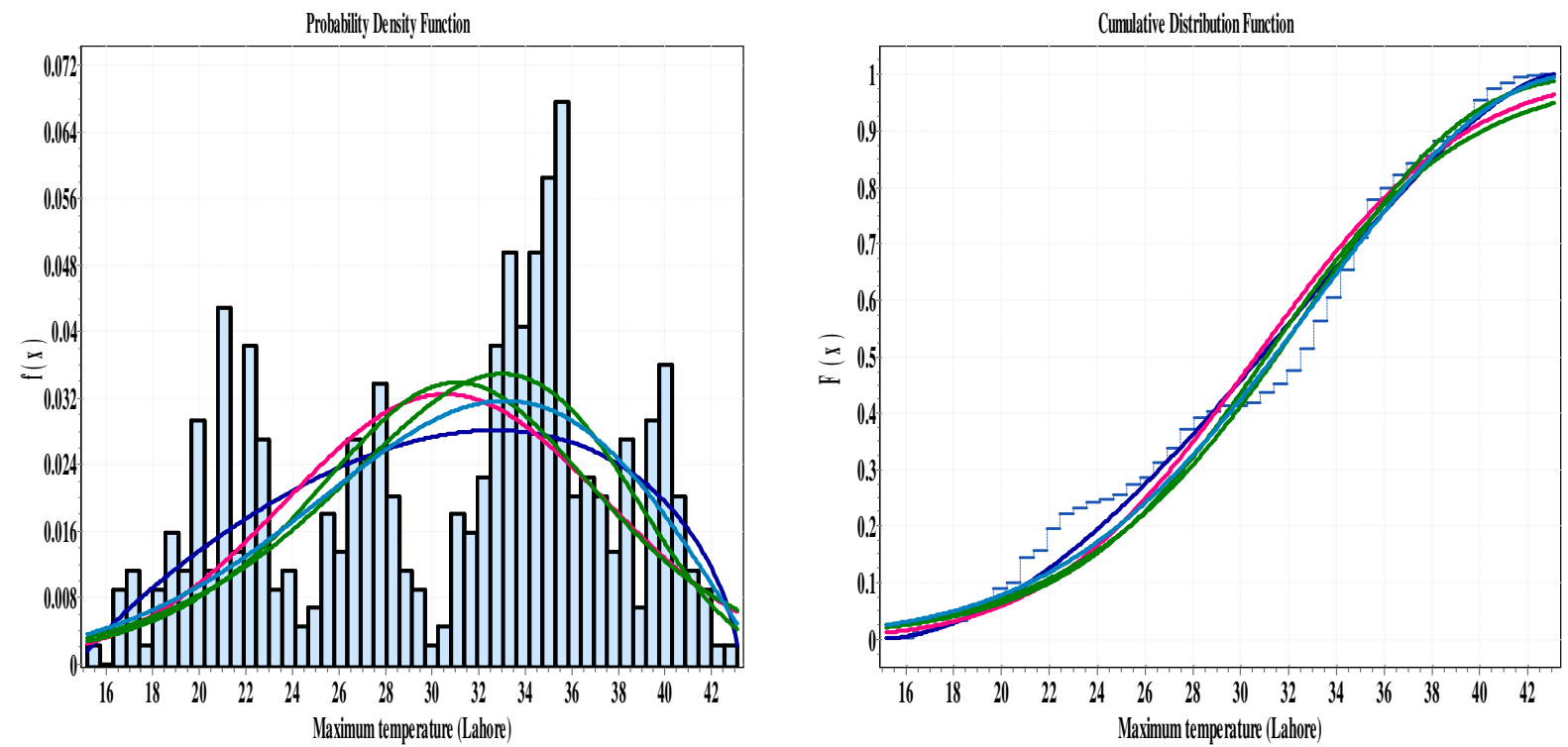

-Kumaraswamy -Inv. Gaussian(3P) - Log.Logistic (3P) -Weibul (3P)
-Gen.Extreme Value

(c)
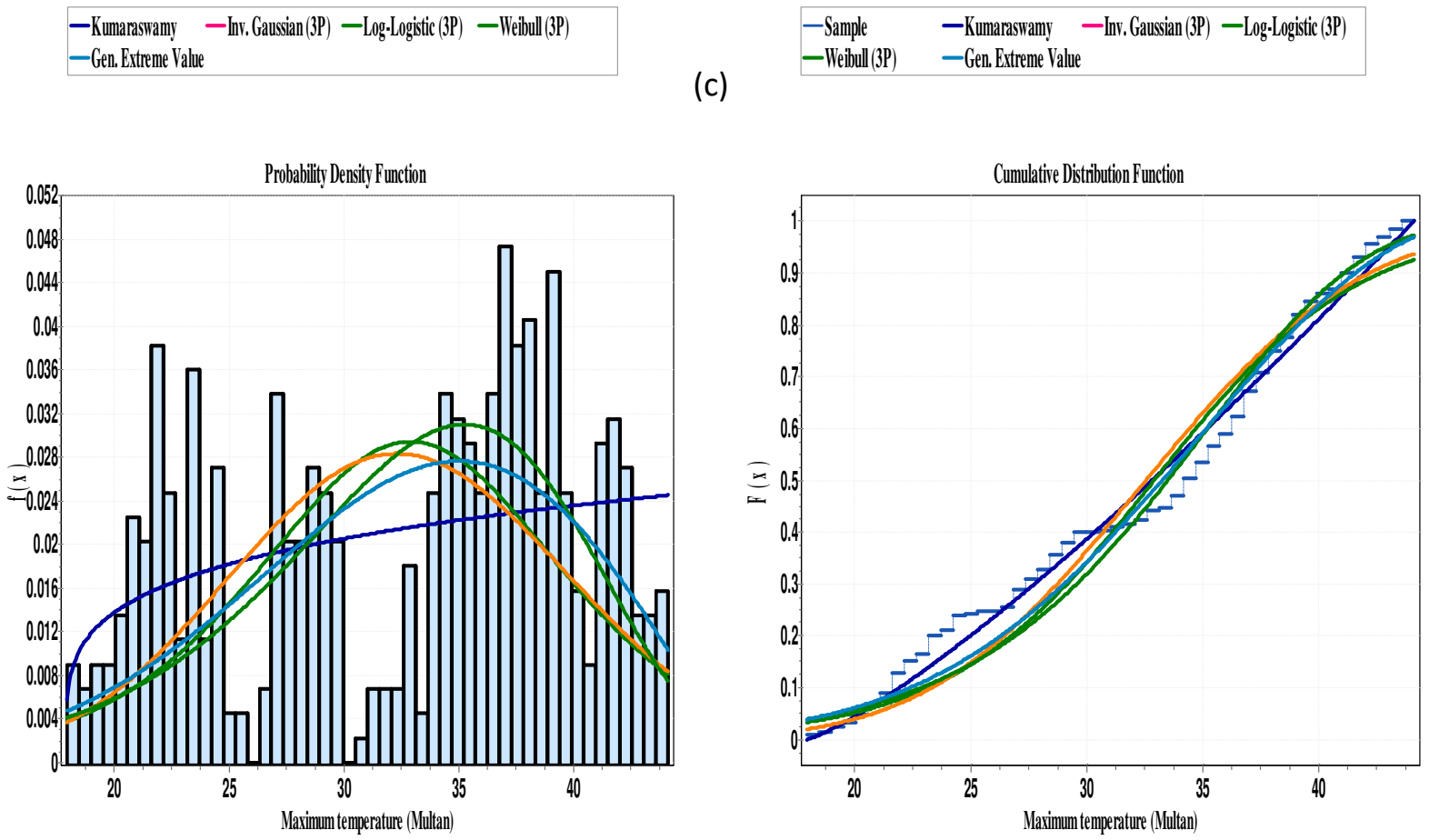

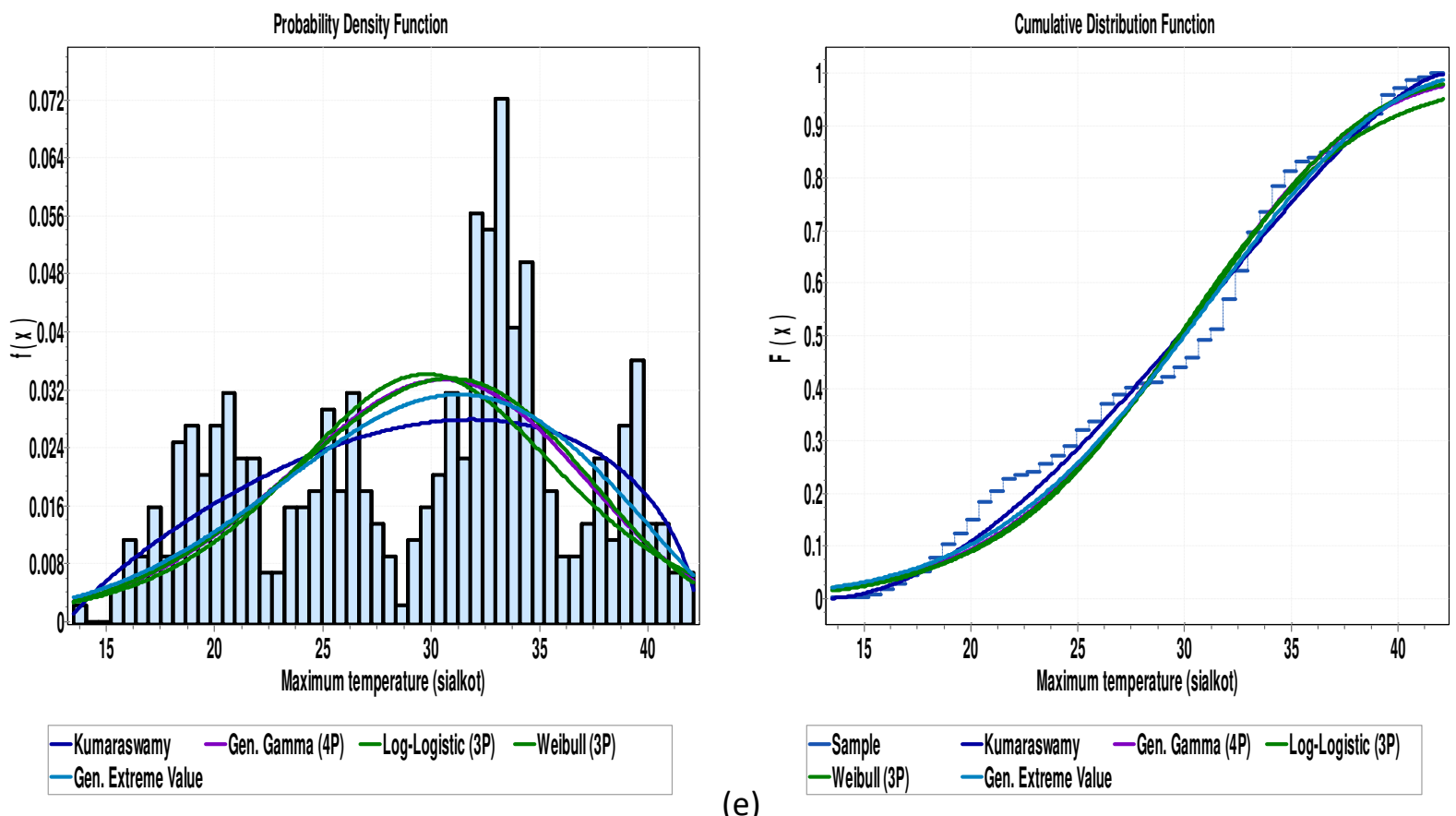

(e)

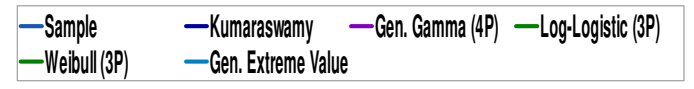

Fig.2 Histograms with fitted distributions and cumulative distribution functions for: (a)

4 Hyderabad (b) Karachi (c) Lahore (d) Multan and (e) Sialkot

9 Table. Probability distributions (PDF) and cumulative distribution (CDF) for each distribution.

\begin{tabular}{|l|c|c|}
\hline Distributions & PDF & CDF \\
\hline $\begin{array}{l}\text { Exponential (2P) } \\
\text { Gormal }\end{array}$ & $f(x)=\lambda \exp (-\lambda(x-\lambda)$ & $F(x)=1-\exp (-\lambda(x-\gamma))$ \\
Inv. Gaussian & $f(x)=\frac{x^{\alpha-1}}{\beta^{\alpha} \Gamma(\alpha)} \exp (-x / \beta)$ & $F(x)=\frac{\Gamma \mathrm{x} / \beta(\alpha)}{\Gamma(\alpha)}$ \\
& $f(x)=\sqrt{\frac{\lambda}{2 \pi x^{3}} \exp \left(-\frac{\lambda(x-\mu)^{2}}{2 \mu^{2} x}\right)}$ & $F(x)=\varnothing\left(\sqrt{\left.\frac{\lambda}{x}\left(\frac{x}{\mu}-1\right)\right)}\right.$ \\
$\begin{array}{l}\text { Logistic } \\
\text { Log-Logistic 3 }\end{array}$ & $f(x)=\frac{\alpha}{\beta}\left(\frac{x-\gamma}{\beta}\right)^{\alpha-1}\left(1+\left(\frac{x-\gamma}{\beta}\right)^{\alpha}\right)^{-2}$ & $F(x)=\left(1+\left(\frac{\beta}{x}\left(\frac{x}{\mu}+1\right)\right) \exp (2 \lambda / \mu)\right.$ \\
\end{tabular}




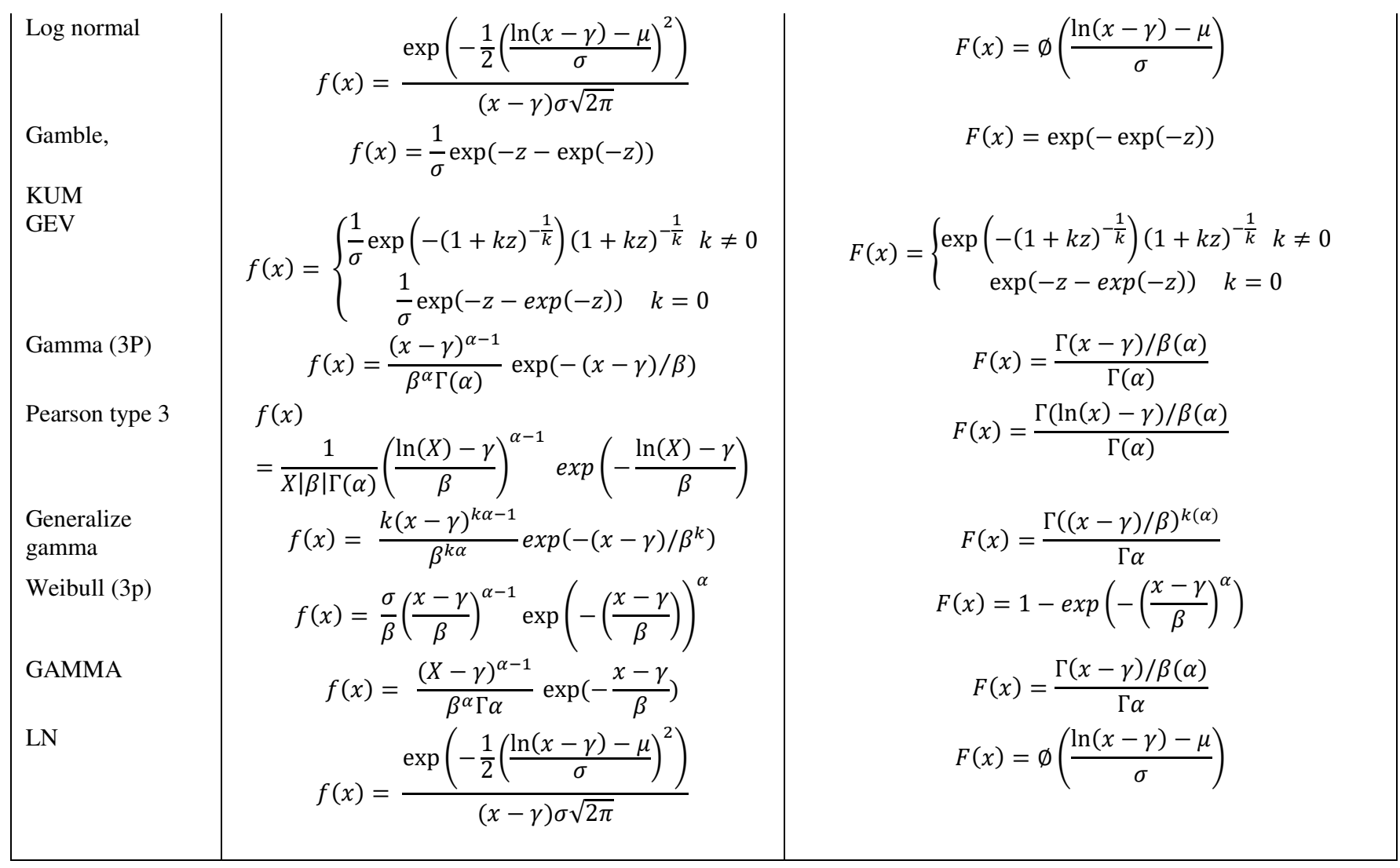

1

2 Table 2. Descriptive analysis of five stations

\begin{tabular}{lcccccccc}
\hline Variable & SD & Skewness & Kurtosis & Mean & $\begin{array}{c}\text { Max } \\
\text { Temp }\end{array}$ & Latitude & $\begin{array}{c}\text { Longitude } \\
\text { Altitude } \\
(\mathrm{m})\end{array}$ \\
\hline Hyderabad & 0.78 & 0.03 & 0.14 & 41.32 & 43.10 & $\mathbf{2 5}^{\circ} \mathbf{2 3}^{\prime}$ & $\mathbf{6 8}^{\circ} \mathbf{2 2} \mathbf{\prime}^{\prime}$ & 7 \\
Karachi & 0.76 & 1.08 & 4.16 & 36.23 & 39 & $24^{\circ} 54^{\prime}$ & $67^{\circ} 4^{\prime}$ & 4 \\
Lahore & 1.25 & -0.46 & 1.25 & 40.30 & 43.10 & $31^{\circ} 33^{\prime}$ & $74^{\circ} 19^{\prime}$ & 215 \\
Multan & 1.06 & -0.26 & -0.61 & 42.32 & 44.10 & $30^{\circ} 11^{\prime}$ & $71^{\circ} 28^{\prime}$ & 123 \\
Sialkot & 1.55 & -0.14 & -0.30 & 40.04 & 43.23 & $32^{\circ} 30^{\prime}$ & $74^{\circ} 31^{\prime}$ & 256 \\
\hline
\end{tabular}

3

Table 3. Achieved statistical indicators of different distribution functions for the selected 5 Stations. 


\begin{tabular}{|c|c|c|c|c|c|c|}
\hline Stations & Models & KS & AD & AIC & BIC & Ranking \\
\hline \multirow[t]{12}{*}{ Hyderabad } & EXP & 0.1624 & 3.9981 & 2776.031 & 2785.011 & 12 \\
\hline & $\mathrm{N}$ & 0.0671 & 0.4159 & 2735.534 & 2744.677 & 3 \\
\hline & IG & 0.06762 & 0.4161 & 2735.999 & 2745.908 & 4 \\
\hline & $\mathrm{L}$ & 0.08922 & 0.7404 & 2755.989 & 2764.908 & 10 \\
\hline & LL3 & 0.07071 & 0.4874 & 2737.679 & 2746.980 & 5 \\
\hline & LN3 & 0.07155 & 0.4541 & 2744.210 & 2752.401 & 7 \\
\hline & GUM & 0.11081 & 1.1564 & 2757.399 & 2765.590 & 11 \\
\hline & KUM & 0.03591 & 0.2765 & 2730.076 & 2741.980 & 1 \\
\hline & GEV & 0.05475 & 0.2988 & 2732.890 & 2743.066 & 2 \\
\hline & Wei3 & 0.08661 & 0.6363 & 2752.534 & 2761.920 & 9 \\
\hline & PE3 & 0.07356 & 0.4596 & 2750.456 & 2759.098 & 8 \\
\hline & GG (4) & 0.0713 & 0.4497 & 2741.089 & 2750.098 & 6 \\
\hline \multirow[t]{12}{*}{ Karachi } & EXP & 0.28726 & 70.427 & 2314.958 & 2323.149 & 12 \\
\hline & $\mathrm{N}$ & 0.1191 & 10.686 & 2259.021 & 2267.032 & 6 \\
\hline & IG & 0.11835 & 10.599 & 2254.753 & 2261.098 & 5 \\
\hline & $\mathrm{L}$ & 0.11953 & 12.821 & 2264.123 & 2272.701 & 7 \\
\hline & LL3 & 0.09089 & 9.2624 & 2252.382 & 2261.890 & 4 \\
\hline & LN3 & 0.12672 & 11.496 & 2265.067 & 2273.032 & 8 \\
\hline & GUM & 0.18769 & 38.955 & 2303.642 & 2311.833 & 11 \\
\hline & KUM & 0.07047 & 3.7466 & 2233.042 & 2241.021 & 2 \\
\hline & GEV & 0.06033 & 10.164 & 2221.620 & 2229.042 & 1 \\
\hline & Wei3 & 0.07542 & 3.6572 & 2235.021 & 2242.324 & 3 \\
\hline & PE3 & 0.13562 & 12.357 & 2279.034 & 2287.435 & 10 \\
\hline & GG (4) & 0.12716 & 11.561 & $2276 . .067$ & 2284.032 & 9 \\
\hline \multirow[t]{12}{*}{ Lahore } & EXP & 0.23172 & 57.062 & 3054.008 & 3067.098 & 12 \\
\hline & $\mathrm{N}$ & 0.13854 & 9.5406 & 3005.043 & 3012.760 & 8 \\
\hline & IG & 0.13415 & 9.6267 & 2954.231 & 2964.098 & 6 \\
\hline & $\mathrm{L}$ & 0.15459 & 13.645 & 3011.890 & 3020.005 & 8 \\
\hline & LL3 & 0.11894 & 9.5841 & 2875.078 & 2885.675 & 4 \\
\hline & LN3 & 0.13616 & 10.041 & 2999.910 & 3008.101 & 7 \\
\hline & GUM & 0.20152 & 29.918 & 3021.575 & 3029.767 & 11 \\
\hline & KUM & 0.0125 & 4.5895 & 2800.981 & 2812.109 & 1 \\
\hline & GEV & 0.0939 & 4.7563 & 2823.081 & $2835.142 \backslash$ & 2 \\
\hline & Wei3 & 0.09681 & 6.4167 & 2856.052 & 2864.160 & 3 \\
\hline & PE3 & 0.15904 & 12.143 & 3017.879 & 3024.009 & 10 \\
\hline & GG (4) & 0.13815 & 9.5734 & 2912.098 & 2922.564 & 5 \\
\hline
\end{tabular}




\begin{tabular}{|c|c|c|c|c|c|c|c|}
\hline \multirow[t]{11}{*}{ Multan } & EXP & 0.21595 & 42.866 & 3075.670 & 3084.003 & 11 & 1 \\
\hline & $\mathrm{N}$ & 0.12852 & 10.988 & 3023.006 & 3031.890 & 6 & \\
\hline & IG & 0.13224 & 11.123 & 3033.045 & 3041.008 & 7 & \\
\hline & $\mathrm{L}$ & 0.14184 & 15.788 & 3041.678 & 3049.014 & 8 & \\
\hline & LL3 & 0.10831 & 10.906 & 3005.352 & 3013.008 & 3 & \\
\hline & LN3 & 0.12375 & 11.209 & 3073.808 & 3082.000 & 5 & \\
\hline & GUM & 0.19464 & 30.893 & 3056.536 & 3063.002 & 10 & \\
\hline & KUM & 0.09133 & 3.7197 & 2956.870 & 2967.054 & 2 & \\
\hline & GEV & 0.08861 & 6.0058 & 2901.065 & 2911.045 & 1 & \\
\hline & Wei3 & 0.10279 & 7.9024 & 2978.076 & 2988.078 & 4 & \\
\hline & PE3 & 0.14867 & 12.568 & 3044.081 & 3052.061 & 9 & \\
\hline \multirow[t]{12}{*}{ Sialkot } & EXP & 0.22985 & 58.414 & 3148.089 & 3153.079 & 12 & \\
\hline & $\mathrm{N}$ & 0.12617 & 7.929 & 3011.484 & 3019.676 & 7 & \\
\hline & IG & 0.12081 & 8.2295 & 2983.072 & 2992.560 & 6 & \\
\hline & $\mathrm{L}$ & 0.14319 & 11.702 & 3042.014 & 3048.019 & 10 & \\
\hline & LL3 & 0.11209 & 8.2 & 2957.546 & 2968.745 & 5 & \\
\hline & LN3 & 0.12929 & 8.3351 & 3032.168 & 3040.360 & 8 & \\
\hline & GUM & 0.18772 & 26.492 & 3134.089 & 3142.190 & 11 & \\
\hline & KUM & 0.09057 & 3.4658 & 2899.043 & 2901.043 & 1 & \\
\hline & GEV & 0.09113 & 4.6078 & 2913.060 & 2920.609 & 2 & \\
\hline & Wei3 & 0.10358 & 6.3469 & 2925.012 & 2932.081 & 3 & \\
\hline & PE3 & 0.14089 & 9.5884 & 3034.073 & 3042.009 & 9 & \\
\hline & GG (4) & 0.10862 & 6.4591 & 2934.053 & 2945.019 & 4 & \\
\hline
\end{tabular}

